\title{
Effective action for superfluid Fermi systems in the strong-coupling limit
}

\author{
N. Dupuis \\ Department of Mathematics, Imperial College, \\ 180 Queen's Gate, London SWr 2AZ, UK \\ and \\ Laboratoire de Physique des Solides, CNRS UMR 8502, \\ Université Paris-Sud, 91405 Orsay, France
}

(Dated: December 20, 2004)

\begin{abstract}
We derive the low-energy effective action for three-dimensional superfluid Fermi systems in the strong-coupling limit, where superfluidity originates from Bose-Einstein condensation of composite bosons. Taking into account density and pairing fluctuations on the same footing, we show that the effective action involves only the fermion density $\rho_{\mathbf{r}}$ and its conjugate variable, the phase $\theta_{\mathbf{r}}$ of the pairing order parameter $\Delta_{\mathbf{r}}$. We recover the standard action of a Bose superfluid of density $\rho_{\mathbf{r}} / 2$, where the bosons have a mass $m_{B}=2 m$ and interact via a repulsive contact potential with amplitude $g_{B}=4 \pi a_{B} / m_{B}, a_{B}=2 a$ ( $a$ the s-wave scattering length associated to the fermionfermion interaction in vacuum). For lattice models, the derivation of the effective action is based on the mapping of the attractive Hubbard model onto the Heisenberg model in a uniform magnetic field, and a coherent state path integral representation of the partition function. The effective description of the Fermi superfluid in the strong-coupling limit is a Bose-Hubbard model with an intersite hopping amplitude $t_{B}=J / 2$ and an on-site repulsive interaction $U_{B}=2 J z$, where $J=4 t^{2} / U(t$ and $-U$ are the intersite hopping amplitude and the on-site attraction in the (fermionic) Hubbard model, $z$ the number of nearest-neighbor sites).

PACS numbers: 74.20.Fg, 71.10.Fd, 05.30.Jp
\end{abstract}

\section{INTRODUCTION}

Recent progress in the experimental control of ultracold atomic Fermi ${ }^{1.2 .3 .4 .5}$ gases has revived the interest in the crossover from the weak-coupling BCS limit of superfluid fermions to the strong-coupling limit of condensed composite bosons ${ }^{6.7}$ In this paper, we derive the lowenergy effective action for a superfluid Fermi system in the strong-coupling limit, both in continuum and lattice models. The latter may be relevant for high- $T_{c}$ superconductors or ultracold Fermi gases in an optical lattice.

A Bose superfluid is described by a complex field $\psi_{B \mathbf{r}}=\sqrt{\rho_{B \mathbf{r}}} e^{i \theta_{B \mathbf{r}}}$ where $\rho_{B \mathbf{r}}$ is the boson density at position $\mathbf{r}$ in space. The equation of motion derived from the standard action of a Bose system leads to the GrossPitaevskii equation $\stackrel{8.9}{,}$ i.e. a non-linear Schrödinger equation for the $\psi_{B}$ field. The Gross-Pitaevskii equation yields a simple description of quantum macroscopic phenomena like the Josephson effect or the flux quantization, 10.11 and has proven to be a tool of choice for the understanding of many phenomena in ultracold atomic Bose gases. ${ }^{12}$ In Fermi systems, there is in general no simple relation between the amplitude of the superfluid (pairing) order parameter $\Delta_{\mathrm{r}}$ and the fermion density $\rho_{\mathbf{r}}$. This suggests that a minimal description, aiming at making contact with the standard description of a Bose superfluid, should at least include the superfluid order parameter $\Delta_{\mathbf{r}}$ and the density $\rho_{\mathbf{r}}$ from the outset. In the strong-coupling limit, where superfluidity originates from Bose-Einstein condensation (BEC) of composite bosons, we expect the description in terms of $\rho_{\mathbf{r}}$ and $\Delta_{\mathbf{r}}=\left|\Delta_{\mathbf{r}}\right| e^{i \theta_{\mathbf{r}}}$ to be redundant and the superfluid to be described by a single complex field $\psi_{\mathbf{r}}=\sqrt{\rho_{\mathbf{r}} / 2} e^{i \theta_{\mathbf{r}}}$ ( $\rho_{\mathbf{r}} / 2$ being the density of composite bosons).

Previous studies of the BCS-BEC crossover in superfluid Fermi systems can be divided into two categories. In the first type of approach, ${ }^{6.13 .14 .15 .16 .17 .18}$ the density $\rho_{\mathbf{r}}$ is not considered explicitely and a pairing field $\Delta_{\mathbf{r}}^{\mathrm{HS}}$ is introduced by means of a Hubbard-Stratonovich transformation of the fermion-fermion interaction. In the BEC limit, the standard action $S\left[\psi^{*}, \psi\right]$ of a Bose superfluid is recovered if one identifies $\psi_{\mathbf{r}}$ to $\Delta_{\mathbf{r}}^{\mathrm{HS}}$ (after a proper rescaling). For a continuum model, the bosons have a mass $m_{B}=2 m$ and interact via a repulsive contact potential with amplitude $g_{B}=4 \pi a_{B} / m_{B}, a_{B}=2 a$ ( $a$ is the swave scattering length associated to the fermion-fermion interaction in vacuum). The main (conceptual) difficulty of this approach is that the Hubbard-Stratonovich field $\Delta_{\mathbf{r}}^{\mathrm{HS}}$ is not the physical pairing field $\Delta_{\mathbf{r}}=\left|\Delta_{\mathbf{r}}\right| e^{i \theta_{\mathbf{r}}}$ but rather its conjugate field ${ }^{19}$ Although both fields coincide at the mean-field level, they differ when fluctuations are taken into account. As a result, $\psi_{\mathbf{r}} \propto \Delta_{\mathbf{r}}^{\mathrm{HS}}$ does not correspond to $\sqrt{\rho_{\mathbf{r}} / 2} e^{i \theta_{\mathbf{r}}}$ as expected.

In the second type of approach to the BCS-BEC crossover 19.20 .21 the physical density and pairing fields, $\rho_{\mathbf{r}}$ and $\Delta_{\mathbf{r}}$, are introduced from the outset. For continuum models, only the weak-coupling limit has been considered ${ }^{19.21}$ For lattice (Hubbard) models in the strong-coupling low-density limit, one finds that the order parameter amplitude and the density are tied by the relation $\left|\Delta_{\mathbf{r}}\right|=\sqrt{\rho_{\mathbf{r}} / 2}$, so that the low-energy effective action can be written in terms of a single complex field, $\Delta_{\mathbf{r}}=\sqrt{\rho_{\mathbf{r}} / 2} e^{i \theta_{\mathbf{r}}} \equiv \psi_{\mathbf{r}}{ }^{19.20}$ In the continuum limit, one finds that the (composite) bosons have a mass $m_{B}=1 / J$ and interact via a repulsive contact potential with ampli- 
tude $g_{B}=8 J$ (in two dimensions), where $J=4 t^{2} / U$ ( $t$ being the intersite hopping amplitude and $-U(U \geq 0)$ the on-site attractive interaction) 20

Most of the theoretical works on the BCS-BEC crossover in ultracold atomic Fermi gases have been formulated within a fermion-boson model, 22 aiming at incorporating the molecular states involved in the Feshbach resonance which drives the crossover. While the equivalence of the fermion-boson model to an effective single-channel model in the crossover region may be questionable $\stackrel{23.24}{ }^{2}$ both models are equivalent in the strong-coupling limit.

The outline of the paper is as follows. In Sec. II we extend the approach of Ref. 19 to the strong-coupling limit of a continuum model. The particle-particle and particle-hole channels are considered on the same footing, and the (physical) density $\left(\rho_{\mathbf{r}}\right)$ and pairing $\left(\Delta_{\mathbf{r}}\right)$ fields are introduced from the outset. The low-energy effective action is derived by assuming small fluctuations of the collective fields about their mean-field values. We find that fluctuations of $\rho_{\mathbf{r}}$ and $\Delta_{\mathbf{r}}$ are not independent, so that the low-energy action can be written in term of a single complex field $\psi_{\mathbf{r}}=\sqrt{\rho_{\mathbf{r}} / 2} e^{i \theta_{\mathbf{r}}}$. We recover the standard action of a Bose superfluid with $m_{B}=2 \mathrm{~m}$ and $g_{B}=4 \pi a_{B} / m_{B}, a_{B}=2 a$. For a lattice model (Sec. III), we follow the approach introduced in Ref. 20. We map the attractive Hubbard model onto the half-filled repulsive Hubbard model in a uniform magnetic field coupled to the fermion spins. In the strong-coupling limit, the latter reduces to the Heisenberg model in a uniform field. The low-energy effective action of the attractive model is finally deduced from the coherent state path integral representation of the Heisenberg model. In the low-density limit, where the Pauli principle (which prevents two composite bosons to occupy the same site) should not matter, $\left|\Delta_{\mathbf{r}}\right| \simeq \sqrt{\rho_{\mathbf{r}} / 2}$ and the superfluid Fermi system can be described by the complex field $\psi_{\mathbf{r}}=\sqrt{\rho_{\mathbf{r}} / 2} e^{i \theta_{\mathbf{r}}}$. We find that the effective description of the Fermi superfluid is a Bose-Hubbard model with intersite hopping amplitude $t_{B}=J / 2$ and an on-site repulsive interaction $U_{B}=2 J z$ (where $z$ is the number of nearest-neighbor sites).

\section{CONTINUUM MODEL}

We consider a three-dimensional superfluid fermion system with the action $S=S_{0}+S_{\mathrm{int}}$,

$$
\begin{aligned}
S_{0} & =\int_{0}^{\beta} d \tau \int d^{3} \mathbf{r} c_{\mathbf{r}}^{\dagger}\left(\partial_{\tau}-\mu-\frac{\nabla^{2}}{2 m}\right) c_{\mathbf{r}}, \\
S_{\text {int }} & =-g \int_{0}^{\beta} d \tau \int d^{3} \mathbf{r} c_{\mathbf{r} \uparrow}^{*} c_{\mathbf{r} \downarrow}^{*} c_{\mathbf{r} \downarrow} c_{\mathbf{r} \uparrow},
\end{aligned}
$$

where $c_{\mathbf{r} \sigma}^{(*)}$ are Grassmann variables, $c_{\mathbf{r}}=\left(c_{\mathbf{r} \uparrow}, c_{\mathbf{r} \downarrow}\right)^{T}, \tau$ an imaginary time and $\beta=1 / T$ the inverse temperature. $-g$ is the attractive interaction between fermions $(g \geq 0)$. The chemical potential $\mu$ fixes the average fermion density $\rho_{0}$. To suppress ultraviolet divergences appearing in the perturbation theory, one regularizes ${ }^{15}$ the local fermion-fermion interaction with a cutoff $\Lambda$ acting on the fermion dispersion: $\epsilon_{\mathbf{k}}=|\mathbf{k}|^{2} / 2 m \leq \Lambda . \quad g$ and $\Lambda$ determine the s-wave scattering length $a$ defined by the low-energy limit of the two-body problem in vacuum,

$$
\frac{m}{4 \pi a}=-\frac{1}{g}+\int_{\epsilon_{\mathbf{k}} \leq \Lambda} \frac{d^{3} \mathbf{k}}{(2 \pi)^{3}} \frac{1}{2 \epsilon_{\mathbf{k}}} .
$$

$a$ is negative for small $g$ and diverges when $g=2 \pi^{2} / m \Lambda$. For $g>2 \pi^{2} / m \Lambda$, there is a two-body bound-state (composite boson) with energy $E_{B}=-1 / m a^{2}$ and the scattering length $a$ is positive. The latter also determines the extension of the bound-state. Low-energy properties depend solely on $a$ (and not $g$ or $\Lambda$ ); we shall therefore take the limit $g \rightarrow 0$ and $\Lambda \rightarrow \infty$ with $a$ fixed. In the following, we consider the BEC limit defined by $\rho_{0} a^{3} \ll 1$ $(a>0)$, where superfluidity originates from BEC of composite bosons.

The (real) density and (complex) pairing fields,

$$
\begin{aligned}
\rho_{\mathbf{r}} & =c_{\mathbf{r}}^{\dagger} c_{\mathbf{r}}, \\
S_{\mathbf{r}}^{z} & =c_{\mathbf{r}}^{\dagger} \sigma^{z} c_{\mathbf{r}}, \\
\Delta_{\mathbf{r}} & =c_{\mathbf{r} \downarrow} c_{\mathbf{r} \uparrow}, \\
\Delta_{\mathbf{r}}^{*} & =c_{\mathbf{r} \uparrow}^{*} c_{\mathbf{r} \downarrow}^{*},
\end{aligned}
$$

can be introduced in the action by means of real $\left(\rho_{\mathbf{r}}^{\mathrm{HS}}, \tilde{\rho}_{\mathbf{r}}^{\mathrm{HS}}\right)$ and complex $\left(\Delta_{\mathbf{r}}^{\mathrm{HS}}\right)$ Lagrange multipliers:

$$
\begin{aligned}
S= & \int_{0}^{\beta} d \tau \int d^{3} \mathbf{r}\left\{c_{\mathbf{r}}^{\dagger}\left(\partial_{\tau}-\mu-\frac{\nabla^{2}}{2 m}\right) c_{\mathbf{r}}\right. \\
& -g \gamma\left|\Delta_{\mathbf{r}}\right|^{2}-\frac{g \alpha}{4}\left(\rho_{\mathbf{r}}^{2}-S_{\mathbf{r}}^{z^{2}}\right) \\
& +i \rho_{\mathbf{r}}^{\mathrm{HS}}\left(\rho_{\mathbf{r}}-c_{\mathbf{r}}^{\dagger} c_{\mathbf{r}}\right)+i \tilde{\rho}_{\mathbf{r}}^{\mathrm{HS}}\left(S_{\mathbf{r}}^{z}-c_{\mathbf{r}}^{\dagger} \sigma^{z} c_{\mathbf{r}}\right) \\
& \left.+i\left[\Delta_{\mathbf{r}}^{\mathrm{HS}}\left(\Delta_{\mathbf{r}}^{*}-c_{\mathbf{r} \uparrow}^{*} c_{\mathbf{r} \downarrow}^{*}\right)+\text { c.c. }\right]\right\} .
\end{aligned}
$$

$\left(\sigma^{x}, \sigma^{y}, \sigma^{z}\right)$ denotes the Pauli matrices. Integrating over $\rho_{\mathbf{r}}^{\mathrm{HS}}, \tilde{\rho}_{\mathbf{r}}^{\mathrm{HS}}, \Delta_{\mathbf{r}}^{\mathrm{HS}}$ and $\rho_{\mathbf{r}}, S_{\mathbf{r}}^{z}, \Delta_{\mathbf{r}}$, we recover the original action (1) if we choose $\alpha+\gamma=1{ }^{25}$ The relative weights $\alpha$ and $\gamma$ of the particle-hole and particle-particle channels are arbitrary. All the resulting effective actions are equivalent when treated exactly. However, to recover the mean-field results from a saddle-point approximation, we take $\alpha=\gamma=1$. When only low-energy long-wavelength fluctuations about the mean-field state are considered, there is no overlapping of the two channels and therefore no overcounting. ${ }^{19}$ Note that by integrating out the physical fields $S_{\mathbf{r}}^{z}, \rho_{\mathbf{r}}$ and $\Delta_{\mathbf{r}}$, one recovers the action $S\left[c, \rho_{\mathbf{r}}^{\mathrm{HS}}, \tilde{\rho}_{\mathbf{r}}^{\mathrm{HS}}, \Delta_{\mathbf{r}}^{\mathrm{HS}}\right]$ which is generally obtained by means of a Hubbard-Stratonovich decoupling of the interaction term. Thus the Lagrange multipliers $\rho_{\mathbf{r}}^{\mathrm{HS}}, \tilde{\rho}_{\mathbf{r}}^{\mathrm{HS}}$ and $\Delta_{\mathbf{r}}^{\mathrm{HS}}$ enforcing the constraints (3) can also be seen as HubbardStratonovich fields $\frac{19}{9}$ In the following, we neglect spin fluctuations $\left(\tilde{\rho}_{\mathbf{r}}^{\mathrm{HS}}\right.$ and $\left.S_{\mathbf{r}}^{z}\right)$ since they do not play an important role when the interaction is attractive. 


\section{A. Mean-field theory}

The mean-field theory is obtained from a saddle-point approximation where the fields $\rho_{\mathbf{r}}, \Delta_{\mathbf{r}}, \rho_{\mathbf{r}}^{\mathrm{HS}}$ and $\Delta_{\mathbf{r}}^{\mathrm{HS}}$ are taken space and time independent. The saddle-point equations read

$$
\begin{array}{cl}
\rho_{0}=\left\langle c_{\mathbf{r}}^{\dagger} c_{\mathbf{r}}\right\rangle, & i \rho_{0}^{\mathrm{HS}}=\frac{g}{2} \rho_{0}, \\
\Delta_{0}=\left\langle c_{\mathbf{r} \downarrow} c_{\mathbf{r} \uparrow}\right\rangle, & i \Delta_{0}^{\mathrm{HS}}=g \Delta_{0}, \\
\Delta_{0}^{*}=\left\langle c_{\mathbf{r} \uparrow}^{*} c_{\mathbf{r} \downarrow}^{*}\right\rangle, & i \Delta_{0}^{\mathrm{HS}}{ }^{*}=g \Delta_{0}^{*} .
\end{array}
$$

With no loss of generality, we can take $\Delta_{0}=\Delta_{0}^{*}$ real. $i \Delta_{0}^{\mathrm{HS}}=i \Delta_{0}^{\mathrm{HS}}{ }^{*}$ is then real at the saddle point. It is convenient to redefine $i \Delta_{0}^{\mathrm{HS}} \rightarrow \Delta_{0}^{\mathrm{HS}}$ and $i \Delta_{0}^{\mathrm{HS}}{ }^{*} \rightarrow \Delta_{0}^{\mathrm{HS}}{ }^{*}$ (so that $\Delta_{0}^{\mathrm{HS}}=\Delta_{0}^{\mathrm{HS}}{ }^{*}$ is real) and absorb $i \rho_{0}^{\mathrm{HS}}$ in the definition of the chemical potential. The mean-field action is then (up to an additive constant)

$$
\begin{aligned}
S_{\mathrm{MF}}= & \int_{0}^{\beta} d \tau \int d^{3} \mathbf{r}\left[c_{\mathbf{r}}^{\dagger}\left(\partial_{\tau}-\mu-\frac{\nabla^{2}}{2 m}\right) c_{\mathbf{r}}\right. \\
& \left.-\Delta_{0}^{\mathrm{HS}}\left(c_{\mathbf{r} \uparrow}^{*} c_{\mathbf{r} \downarrow}^{*}+\text { c.c. }\right)\right] .
\end{aligned}
$$

From (6), we readily obtain the normal and anomalous Green functions,

$$
\begin{gathered}
G(\mathbf{k}, i \omega)=-\left\langle c_{\sigma}(\mathbf{k}, i \omega) c_{\sigma}^{*}(\mathbf{k}, i \omega)\right\rangle=\frac{-i \omega-\xi_{\mathbf{k}}}{\omega^{2}+E_{\mathbf{k}}^{2}} \\
F(\mathbf{k}, i \omega)=-\left\langle c_{\sigma}(\mathbf{k}, i \omega) c_{\bar{\sigma}}(-\mathbf{k},-i \omega)\right\rangle=\frac{\Delta_{0}^{\mathrm{HS}}}{\omega^{2}+E_{\mathbf{k}}^{2}} \\
F^{*}(\mathbf{k}, i \omega)=-\left\langle c_{\bar{\sigma}}^{*}(-\mathbf{k},-i \omega) c_{\sigma}^{*}(\mathbf{k}, i \omega)\right\rangle=F(\mathbf{k}, i \omega),
\end{gathered}
$$

where $E_{\mathbf{k}}=\left(\xi_{\mathbf{k}}^{2}+\Delta_{0}^{\mathrm{HS}^{2}}\right)^{1 / 2}, \xi_{\mathbf{k}}=\epsilon_{\mathbf{k}}-\mu$, and $\bar{\sigma}=-\sigma$. $c_{\sigma}(\mathbf{k}, i \omega)$ is the Fourier transformed field of $c_{\mathbf{r} \sigma}$ and $\omega$ a fermionic Matsubara frequency. Using (2) and (7), we can rewrite the saddle-point equations (5) as

$$
\begin{aligned}
\frac{m}{4 \pi a} & =\int_{\mathbf{k}}\left(\frac{1}{2 \epsilon_{\mathbf{k}}}-\frac{1}{2 E_{\mathbf{k}}}\right), \\
\rho_{0} & =\int_{\mathbf{k}}\left(1-\frac{\xi_{\mathbf{k}}}{E_{\mathbf{k}}}\right)
\end{aligned}
$$

where $\int_{\mathbf{k}} \equiv \int d^{3} \mathbf{k} /(2 \pi)^{3}$. Eqs. (8) determine the chemical potential $\mu$ and the order parameter $\Delta_{0}^{\mathrm{HS}}=g \Delta_{0}$. In the strong-coupling limit $\rho_{0} a^{3} \ll 1$, one obtains (see Appendix B]

$$
\begin{aligned}
\mu & =-\frac{1}{2 m a^{2}}\left(1-2 \pi \rho_{0} a^{3}\right), \\
\Delta_{0}^{\mathrm{HS}} & =\left(\frac{4 \pi \rho_{0}}{m^{2} a}\right)^{1 / 2}\left(1+\frac{\pi}{4} \rho_{0} a^{3}\right) .
\end{aligned}
$$

\section{B. Low-energy effective action}

In this section, we derive the low-energy effective action for the physical fields $\rho_{\mathbf{r}}$ and $\Delta_{\mathbf{r}}$. Since our derivation partially follows Ref. 19, we describe only the main steps (technical details are given in Appendix A). The main assumption is that the collective bosonic fields $\rho_{\mathbf{r}}$, $\rho_{\mathbf{r}}^{\mathrm{HS}}, \Delta_{\mathbf{r}}$ and $\Delta_{\mathbf{r}}^{\mathrm{HS}}$ weakly fluctuate about their mean-field values.

Starting from the action (4) (with $\alpha=\gamma=1$ ), where

$$
\Delta_{\mathbf{r}}=\left|\Delta_{\mathbf{r}}\right| e^{i \theta_{\mathbf{r}}}
$$

we perform the change of variables

$$
c_{\mathbf{r}} \rightarrow c_{\mathbf{r}} e^{\frac{i}{2} \theta_{\mathbf{r}}}, \quad \Delta_{\mathbf{r}}^{\mathrm{HS}} \rightarrow \Delta_{\mathbf{r}}^{\mathrm{HS}} e^{i \theta_{\mathbf{r}}} .
$$

We then consider the shift $\rho_{\mathbf{r}}^{\mathrm{HS}} \rightarrow \rho_{0}^{\mathrm{HS}}+\rho_{\mathbf{r}}^{\mathrm{HS}}, i \Delta_{\mathbf{r}}^{\mathrm{HS}} \rightarrow$ $\Delta_{0}^{\mathrm{HS}}+i \Delta_{\mathbf{r}}^{\mathrm{HS}}$ and $i \Delta_{\mathbf{r}}^{\mathrm{HS}} \rightarrow \Delta_{0}^{\mathrm{HS}}+i \Delta_{\mathbf{r}}^{\mathrm{HS}}{ }^{*}$ (recall that a factor $i$ has been included in $\Delta_{0}^{\mathrm{HS}}$ and $\Delta_{0}^{\mathrm{HS}}{ }^{*}$ ), so that the Hubbard-Stratonovich fields $\rho_{\mathbf{r}}^{\mathrm{HS}}$ and $\Delta_{\mathbf{r}}^{\mathrm{HS}}$ now describe (small) fluctuations about the mean-field values. This leads to the action

$$
\begin{aligned}
S= & S_{\mathrm{MF}}+\int_{0}^{\beta} d \tau \int d^{3} \mathbf{r}\left[c _ { \mathbf { r } } ^ { \dagger } \left(\frac{i}{2} \dot{\theta}_{\mathbf{r}}-\frac{i}{4 m} \nabla \theta_{\mathbf{r}} \cdot \overleftrightarrow{\nabla}\right.\right. \\
& \left.+\frac{\left(\boldsymbol{\nabla} \theta_{\mathbf{r}}\right)^{2}}{8 m}-\frac{\mu_{B}}{2}-i \rho_{\mathbf{r}}^{\mathrm{HS}}\right) c_{\mathbf{r}}-i\left(\Delta_{\mathbf{r}}^{\mathrm{HS}} c_{\mathbf{r} \uparrow}^{*} c_{\mathbf{r} \downarrow}^{*}+\text { c.c. }\right) \\
& +\left(i \Delta_{\mathbf{r}}^{\mathrm{HS}}+i \Delta_{\mathbf{r}}^{\mathrm{HS}}+2 \Delta_{0}^{\mathrm{HS}}\right)\left|\Delta_{\mathbf{r}}\right| \\
& \left.-g\left|\Delta_{\mathbf{r}}\right|^{2}-\frac{g}{4} \rho_{\mathbf{r}}^{2}+\left(i \rho_{0}^{\mathrm{HS}}+i \rho_{\mathbf{r}}^{\mathrm{HS}}\right) \rho_{\mathbf{r}}\right]
\end{aligned}
$$

where $\overleftrightarrow{\nabla}=\vec{\nabla}-\overleftarrow{\nabla}$. Here we write the chemical potential as $\mu=\mu_{\mathrm{MF}}+\mu_{B} / 2$ where $\mu_{\mathrm{MF}}$ is the chemical potential in the mean-field approximation. The next step is to shift $\rho_{\mathbf{r}}^{\mathrm{HS}}, i \rho_{\mathbf{r}}^{\mathrm{HS}} \rightarrow i \rho_{\mathbf{r}}^{\mathrm{HS}}+i \dot{\theta}_{\mathbf{r}} / 2+\left(\boldsymbol{\nabla} \theta_{\mathbf{r}}\right)^{2} / 8 m-\mu_{B} / 2$, and to introduce Nambu spinors $\phi_{\mathbf{r}}=\left(c_{\mathbf{r} \uparrow}, c_{\mathbf{r} \downarrow}^{*}\right)^{T}$. This gives

$$
\begin{aligned}
S= & S_{\mathrm{MF}}+S^{\prime}+\int_{0}^{\beta} d \tau \int d^{3} \mathbf{r}\left[-g\left|\Delta_{\mathbf{r}}\right|^{2}-\frac{g}{4} \rho_{\mathbf{r}}^{2}\right. \\
& +\left(2 \Delta_{0}^{\mathrm{HS}}+i \Delta_{\mathbf{r}}^{\mathrm{HS}}+i \Delta_{\mathbf{r}}^{\mathrm{HS}}\right)\left|\Delta_{\mathbf{r}}\right| \\
& \left.+\rho_{\mathbf{r}}\left(i \rho_{0}^{\mathrm{HS}}+i \rho_{\mathbf{r}}^{\mathrm{HS}}+\frac{i}{2} \dot{\theta}_{\mathbf{r}}+\frac{\left(\boldsymbol{\nabla} \theta_{\mathbf{r}}\right)^{2}}{8 m}-\frac{\mu_{B}}{2}\right)\right]
\end{aligned}
$$

where

$$
\begin{aligned}
S^{\prime}= & \int_{0}^{\beta} d \tau \int d^{3} \mathbf{r}\left(-i \rho_{\mathbf{r}}^{\mathrm{HS}} j_{0 \mathbf{r}}^{z}+\frac{1}{2} \nabla \theta_{\mathbf{r}} \cdot \mathbf{j}_{\mathbf{r}}^{0}\right. \\
& \left.-i \Delta_{\mathbf{r}}^{\mathrm{HS}} j_{0 \mathbf{r}}^{+}-i \Delta_{\mathbf{r}}^{\mathrm{HS}} j_{0 \mathbf{r}}^{-}\right), \\
j_{0 \mathbf{r}}^{z}= & \phi_{\mathbf{r}}^{\dagger} \tau^{z} \phi_{\mathbf{r}}=c_{\mathbf{r}}^{\dagger} c_{\mathbf{r}}, \\
j_{0 \mathbf{r}}^{+}= & \phi_{\mathbf{r}}^{\dagger} \tau^{+} \phi_{\mathbf{r}}=c_{\mathbf{r} \uparrow}^{*} c_{\mathbf{r} \downarrow}^{*}, \\
j_{0 \mathbf{r}}^{-}= & \phi_{\mathbf{r}}^{\dagger} \tau^{-} \phi_{\mathbf{r}}=c_{\mathbf{r} \downarrow} c_{\mathbf{r} \uparrow}, \\
\mathbf{j}_{\mathbf{r}}^{0}= & -\frac{i}{2 m} \phi_{\mathbf{r}}^{\dagger} \overleftrightarrow{\nabla} \phi_{\mathbf{r}}=-\frac{i}{2 m} c_{\mathbf{r}}^{\dagger} \overleftrightarrow{\nabla} c_{\mathbf{r}} .
\end{aligned}
$$

$\left(\tau_{x}, \tau_{y}, \tau_{z}\right)$ are Pauli matrices acting in Nambu space. The effective action $S\left[\rho, \rho^{\mathrm{HS}}, \Delta, \Delta^{\mathrm{HS}}\right]$ is obtained by integrating out the fermions. To quadratic order in the bosonic fields and their gradient $\left(\partial_{\tau}\right.$ or $\left.\boldsymbol{\nabla}\right)$, it is sufficient to retain the first and second order cumulants of $S^{\prime}$ 
with respect to the mean-field action:

$$
\begin{gathered}
S\left[\rho, \rho^{\mathrm{HS}}, \Delta, \Delta^{\mathrm{HS}}\right]=\left\langle S^{\prime}-\frac{S^{\prime 2}}{2}\right\rangle_{c} \\
+\int_{0}^{\beta} d \tau \int d^{3} \mathbf{r}\left[-g\left|\Delta_{\mathbf{r}}\right|^{2}-\frac{g}{4} \rho_{\mathbf{r}}^{2}\right. \\
+\left(2 \Delta_{0}^{\mathrm{HS}}+i \Delta_{\mathbf{r}}^{\mathrm{HS}}+i \Delta_{\mathbf{r}}^{\mathrm{HS}}\right)\left|\Delta_{\mathbf{r}}\right| \\
\left.+\rho_{\mathbf{r}}\left(i \rho_{0}^{\mathrm{HS}}+i \rho_{\mathbf{r}}^{\mathrm{HS}}+\frac{i}{2} \dot{\theta}_{\mathbf{r}}+\frac{\left(\nabla \theta_{\mathbf{r}}\right)^{2}}{8 m}-\frac{\mu_{B}}{2}\right)\right],
\end{gathered}
$$

where the averages $\langle\cdots\rangle_{c}$ are calculated with respect to the mean-field action $S_{\mathrm{MF}}$. Calculating the first and second order cumulants and integrating out the HubbardStratonovich fields $\rho_{\mathbf{r}}^{\mathrm{HS}}$ and $\Delta_{\mathbf{r}}^{\mathrm{HS}}$ (Appendix $\mathrm{A}$ ), we obtain

$$
\begin{array}{r}
S[\rho, \Delta]=\int_{0}^{\beta} d \tau \int d^{3} \mathbf{r} \rho_{\mathbf{r}}\left(\frac{i}{2} \dot{\theta}_{\mathbf{r}}+\frac{\left(\nabla \theta_{\mathbf{r}}\right)^{2}}{8 m}-\frac{\mu_{B}}{2}\right) \\
+\sum_{q}\left(\delta \rho_{-q}, \delta|\Delta|_{-q}\right)\left(\begin{array}{ll}
\beta_{\mathbf{q}} & -g \alpha_{\mathbf{q}} \\
-g \alpha_{\mathbf{q}} & g^{2} \gamma_{\mathbf{q}}
\end{array}\right)\left(\begin{array}{l}
\delta \rho_{q} \\
\delta|\Delta|_{q}
\end{array}\right),
\end{array}
$$

where $\delta \rho_{q}$ and $\delta|\Delta|_{q}$ are the Fourier transforms of $\delta \rho_{\mathbf{r}}=$ $\rho_{\mathbf{r}}-\rho_{0}$ and $\delta\left|\Delta_{\mathbf{r}}\right|=\left|\Delta_{\mathbf{r}}\right|-\Delta_{0}$, and

$$
\begin{aligned}
\beta_{\mathbf{q}} & =\frac{1}{2} \Pi_{00}^{z z}(\mathbf{q})^{-1}-\frac{g}{4}-\frac{1}{C_{\mathbf{q}}}\left[\Pi_{00}^{z z}(\mathbf{q})^{-1} \Pi_{00}^{z+}(\mathbf{q})\right]^{2} \\
-g \alpha_{\mathbf{q}} & =\frac{1}{C_{q}} \Pi_{00}^{z z}(\mathbf{q})^{-1} \Pi_{00}^{z+}(\mathbf{q}) \\
g^{2} \gamma_{\mathbf{q}} & =-\frac{1}{C_{\mathbf{q}}}-g \\
C_{\mathbf{q}} & =-\Pi_{00}^{+-}(\mathbf{q})-\Pi_{00}^{++}(\mathbf{q})+2 \Pi_{00}^{z z}(\mathbf{q})^{-1} \Pi_{00}^{z+}(\mathbf{q})^{2} .
\end{aligned}
$$

We use the notation $q=\left(\mathbf{q}, i \omega_{\nu}\right)$ and $\sum_{q}=\sum_{\mathbf{q}, \omega_{\nu}}$ where $\omega_{\nu}$ is a bosonic Matsubara frequency. The mean-field correlation function $\Pi_{00}^{\nu \nu^{\prime}}(q)=\left\langle j_{0}^{\nu}(q) j_{0}^{\nu^{\prime}}(-q)\right\rangle_{c}$ is calculated in Appendix $\mathrm{B}$ and $\Pi_{00}^{\nu \nu^{\prime}}(\mathbf{q})=\Pi_{00}^{\nu \nu^{\prime}}\left(\mathbf{q}, \omega_{\nu}=0\right) . j_{0}^{\nu}(q)$ is the Fourier transformed field of $j_{0 \mathbf{r}}^{\nu}$ [Eq. (15)]. Eq. (17) shows that half the fermion density is the conjugate variable of the phase $\theta_{\mathbf{r}}$ of the pairing field. Eqs. (17.18) agree with Eq. (2.3) of Ref. 19 except for the coefficient of $\delta \rho_{-q} \delta|\Delta|_{q}$ which is found to have opposite sign ${ }^{26}$

We now discuss the strong-coupling limit (not considered in Ref. 19). To leading order in $\rho_{0} a^{3}$ and $|\mathbf{q}| a$, we have (Appendix ${ }^{27}$

$$
\begin{aligned}
\alpha_{\mathbf{q}} & =\left(\frac{1}{4 \pi \rho_{0} a^{3}}\right)^{1 / 2}\left(1+\frac{9}{4} \pi \rho_{0} a^{3}+\frac{1}{6}|\mathbf{q}|^{2} a^{2}\right) \\
\beta_{\mathbf{q}} & =\frac{1}{2 \rho_{0} m a^{2}}\left(1+4 \pi \rho_{0} a^{3}+\frac{1}{4}|\mathbf{q}|^{2} a^{2}\right) \\
\gamma_{\mathbf{q}} & =\frac{m}{2 \pi a}\left(1+\frac{3}{2} \pi \rho_{0} a^{3}+\frac{7}{48}|\mathbf{q}|^{2} a^{2}\right) .
\end{aligned}
$$

Denoting by $\lambda_{\mathbf{q}}^{+}$and $\lambda_{\mathbf{q}}^{-}$the two eigenvalues of the fluctuation matrix appearing in (17), we have

$$
\begin{aligned}
& \lambda_{\mathbf{q}}^{+}=\beta_{\mathbf{q}}+g^{2} \frac{\alpha_{\mathbf{q}}{ }^{2}}{\beta_{\mathbf{q}}} \\
& \lambda_{\mathbf{q}}^{-}=g^{2}\left(\gamma_{\mathbf{q}}-\frac{\alpha_{\mathbf{q}}{ }^{2}}{\beta_{\mathbf{q}}}\right)
\end{aligned}
$$

to order $\mathcal{O}\left(g^{2}\right)$. For $g \rightarrow 0$ (at fixed $a$ ), the mode corresponding to the eigenvalue $\lambda_{\mathbf{q}}^{+}$is frozen, which leads to

$$
\frac{\delta\left|\Delta_{\mathbf{r}}\right|}{\delta \rho_{\mathbf{r}}}=\frac{1}{g}\left(\frac{\pi}{\rho_{0} m^{2} a}\right)^{1 / 2} .
$$

Density $\left(\delta \rho_{\mathbf{r}}\right)$ and modulus $\left(\delta\left|\Delta_{\mathbf{r}}\right|\right)$ fluctuations do not fluctuate independently in the low-energy limit but are tied by the relation (21). From (171921), we deduce that the dynamics of the Fermi superfluid is determined by the effective action

$$
\begin{aligned}
S[\rho, \theta]= & \int_{0}^{\beta} d \tau \int d^{3} \mathbf{r}\left[\rho_{\mathbf{r}}\left(\frac{i}{2} \dot{\theta}_{\mathbf{r}}+\frac{\left(\boldsymbol{\nabla} \theta_{\mathbf{r}}\right)^{2}}{8 m}-\frac{\mu_{B}}{2}\right)\right. \\
& \left.+\frac{\pi a}{2 m}\left(\delta \rho_{\mathbf{r}}\right)^{2}+\frac{\left(\boldsymbol{\nabla} \delta \rho_{\mathbf{r}}\right)^{2}}{32 \rho_{0} m}\right]
\end{aligned}
$$

Introducing the bosonic field

$$
\psi_{\mathbf{r}}=\sqrt{\frac{\rho_{\mathbf{r}}}{2}} e^{i \theta_{\mathbf{r}}}
$$

we recover the standard action of a Bose superfluid,

$$
\begin{aligned}
S\left[\psi^{*}, \psi\right]= & \int_{0}^{\beta} d \tau \int d^{3} \mathbf{r}\left[\psi_{\mathbf{r}}^{*}\left(\partial_{\tau}-\mu_{B}-\frac{\nabla^{2}}{2 m_{B}}\right) \psi_{\mathbf{r}}\right. \\
& \left.+\frac{2 \pi a_{B}}{m_{B}}\left(\psi_{\mathbf{r}}^{*} \psi_{\mathbf{r}}-\rho_{0} / 2\right)^{2}\right]
\end{aligned}
$$

where $m_{B}=2 m$ and $a_{B}=2 a$ are the mass and the scattering length of the bosons. The result $a_{B}=2 a$ corresponds to the Born approximation for the bosonboson scattering, while the exact result is $a_{B}=0.6 a{ }^{28}$ Eqs. (24) and (22) are equivalent in the hydrodynamic regime where $\left(\nabla \rho_{\mathbf{r}}\right)^{2} / \rho_{0} \simeq\left(\nabla \rho_{\mathbf{r}}\right)^{2} / \rho_{\mathbf{r}}{ }^{29}$

Thus, we have shown how, by introducing the physical fields $\rho_{\mathbf{r}}$ and $\Delta_{\mathbf{r}}$ from the outset and expanding about the mean-field state in the strong-coupling limit, one obtains the standard action of a Bose superfluid. Our approach should be contrasted with a number of previous works $6.13 .14,15.16 .17 .18$ where only the pairing HubbardStratonovich field $\Delta_{\mathbf{r}}^{\mathrm{HS}}$ is considered and the expansion is carried out about the non-interacting state, which gives the action (24) but for the field $\sqrt{\rho_{\mathbf{r}} / 2} e^{i \theta_{\mathbf{r}}^{\mathrm{HS}}}$ instead of the $\psi$ field defined in (23) 30 


\section{LATTICE MODEL}

In this section, we consider the attractive Hubbard model on a bipartite lattice, with Hamiltonian

$$
H=-t \sum_{\left\langle\mathbf{r}, \mathbf{r}^{\prime}\right\rangle}\left(c_{\mathbf{r}}^{\dagger} c_{\mathbf{r}^{\prime}}+\text { h.c. }\right)-\mu \sum_{\mathbf{r}} c_{\mathbf{r}}^{\dagger} c_{\mathbf{r}}-U \sum_{\mathbf{r}} n_{\mathbf{r} \uparrow} n_{\mathbf{r} \downarrow} \text {. }
$$

The operator $c_{\mathbf{r} \sigma}^{\dagger}\left(c_{\mathbf{r} \sigma}\right)$ creates (annihilates) a fermion with spin $\sigma$ at the lattice site $\mathbf{r}, c_{\mathbf{r}}=\left(c_{\mathbf{r} \uparrow}, c_{\mathbf{r} \downarrow}\right)^{T}$, and $n_{\mathbf{r} \sigma}=c_{\mathbf{r} \sigma}^{\dagger} c_{\mathbf{r} \sigma} .\left\langle\mathbf{r}, \mathbf{r}^{\prime}\right\rangle$ denotes nearest-neighbor sites. The chemical potential $\mu$ fixes the average density $\rho_{0}$ (i.e. the average number of fermions per site) and $-U(U \geq 0)$ is the on-site attractive interaction.

We are interested in the strong-coupling limit $U \gg$ $t$ where fermions form tightly bound composite bosons which behave as local pairs. The latter Bose condense at low temperature giving rise to superfluidity. In order to derive the low-energy effective action, we could follow the procedure used in Sec. III Here, we shall use a different method, based on the mapping of the attractive Hubbard model in the strong-coupling limit onto the Heisenberg model in a uniform magnetic field ${ }^{20}$ Thus this approach is based on a $t / U$ expansion about the $t=0$ limit rather than on an expansion about the mean-field state. ${ }^{31}$

Under the canonical particle-hole transformation ${ }^{32}$

$$
c_{\mathbf{r} \downarrow} \rightarrow(-1)^{\mathbf{r}} c_{\mathbf{r} \downarrow}^{\dagger}, \quad c_{\mathbf{r} \downarrow}^{\dagger} \rightarrow(-1)^{\mathbf{r}} c_{\mathbf{r} \downarrow}
$$

the Hamiltonian becomes (omitting a constant term)

$$
\begin{aligned}
H= & -t \sum_{\left\langle\mathbf{r}, \mathbf{r}^{\prime}\right\rangle}\left(c_{\mathbf{r}}^{\dagger} c_{\mathbf{r}^{\prime}}+\text { h.c. }\right)-\sum_{\mathbf{r}} c_{\mathbf{r}}^{\dagger}\left(\frac{U}{2}+h_{0} \sigma^{z}\right) c_{\mathbf{r}} \\
& +U \sum_{\mathbf{r}} n_{\mathbf{r} \uparrow} n_{\mathbf{r} \downarrow},
\end{aligned}
$$

and corresponds now to the repulsive Hubbard model in a magnetic field $\mathbf{h}_{0}=h_{0} \hat{\mathbf{z}}$ along the $z$ axis,

$$
h_{0}=\mu+\frac{U}{2}
$$

coupled to the fermion spins. The chemical potential $U / 2$ in (27), together with particle-hole symmetry, implies that the system is half-filled. The density and pairing operators transform into the three components of the spin density operator:

$$
\begin{gathered}
\rho_{\mathbf{r}}=c_{\mathbf{r}}^{\dagger} c_{\mathbf{r}} \rightarrow c_{\mathbf{r}}^{\dagger} \sigma^{z} c_{\mathbf{r}}+1, \\
\Delta_{\mathbf{r}}=c_{\mathbf{r} \downarrow} c_{\mathbf{r} \uparrow} \rightarrow(-1)^{\mathbf{r}} c_{\mathbf{r} \downarrow}^{\dagger} c_{\mathbf{r} \uparrow}, \\
\Delta_{\mathbf{r}}^{\dagger}=c_{\mathbf{r} \uparrow}^{\dagger} c_{\mathbf{r} \downarrow}^{\dagger} \rightarrow(-1)^{\mathbf{r}} c_{\mathbf{r} \uparrow}^{\dagger} c_{\mathbf{r} \downarrow} .
\end{gathered}
$$

The equation fixing $\mu,\left\langle c_{\mathbf{r}}^{\dagger} c_{\mathbf{r}}\right\rangle=\rho_{0}$, becomes an equation fixing the magnetic field: $\left\langle c_{\mathbf{r}}^{\dagger} \sigma^{z} c_{\mathbf{r}}\right\rangle=\rho_{0}-1$.

In the strong-coupling limit $U \gg t$, the Hamiltonian (27) simplifies into ${ }^{20}$

$$
H=J \sum_{\left\langle\mathbf{r}, \mathbf{r}^{\prime}\right\rangle} \mathbf{S}_{\mathbf{r}} \cdot \mathbf{S}_{\mathbf{r}^{\prime}}-2 \mathbf{h}_{0} \cdot \sum_{\mathbf{r}} \mathbf{S}_{\mathbf{r}}
$$

where $J=4 t^{2} / U$ and $\mathbf{S}_{\mathbf{r}}$ is a spin- $\frac{1}{2}$ operator. Using spin- $\frac{1}{2}$ coherent states $\left|\boldsymbol{\Omega}_{\mathbf{r}}\right\rangle\left(\boldsymbol{\Omega}_{\mathbf{r}}^{2}=1\right),{ }^{33}$ the action of the Heisenberg model (30) can be written as

$S[\boldsymbol{\Omega}]=\int_{0}^{\beta} d \tau\left[\sum_{\mathbf{r}}\left[\left\langle\boldsymbol{\Omega}_{\mathbf{r}} \mid \dot{\boldsymbol{\Omega}}_{\mathbf{r}}\right\rangle-\mathbf{h}_{0} \cdot \boldsymbol{\Omega}_{\mathbf{r}}\right]+J \sum_{\left\langle\mathbf{r}, \mathbf{r}^{\prime}\right\rangle} \frac{\boldsymbol{\Omega}_{\mathbf{r}} \cdot \boldsymbol{\Omega}_{\mathbf{r}^{\prime}}}{4}\right]$,

where $\left|\dot{\boldsymbol{\Omega}}_{\mathbf{r}}\right\rangle=\partial_{\tau}\left|\boldsymbol{\Omega}_{\mathbf{r}}\right\rangle$.

The effective action $S[\rho, \Delta]$ of the superfluid system is obtained by rewriting the action (31) in terms of the density and pairing fields of the attractive model. In the strong-coupling limit, Eqs. (29) (written now for fields rather than operators) become ${ }^{20}$

$$
\begin{aligned}
\rho_{\mathbf{r}} & =\Omega_{\mathbf{r}}^{z}+1 \\
\Delta_{\mathbf{r}} & =\frac{(-1)^{\mathbf{r}}}{2} \Omega_{\mathbf{r}}^{-} \\
\Delta_{\mathbf{r}}^{*} & =\frac{(-1)^{\mathbf{r}}}{2} \Omega_{\mathbf{r}}^{+}
\end{aligned}
$$

where $\Omega_{\mathbf{r}}^{ \pm}=\Omega_{\mathbf{r}}^{x} \pm i \Omega_{\mathbf{r}}^{y}$. The condition $\Omega_{\mathbf{r}}^{2}=1$ implies that $\rho_{\mathbf{r}}$ and $\Delta_{\mathbf{r}}$ do not fluctuate independently but are tied by the relation

$$
\left|\Delta_{\mathbf{r}}\right|=\frac{1}{2}\left[\rho_{\mathbf{r}}\left(2-\rho_{\mathbf{r}}\right)\right]^{1 / 2}
$$

In the low-density limit $\left(\rho_{\mathbf{r}} \ll 1\right)$, where the Pauli principle (which prevents two composite bosons to occupy the same site) should not matter, we expect to recover the standard action of a Bose superfluid. In that limit, $\left|\Delta_{\mathbf{r}}\right| \simeq \sqrt{\rho_{\mathbf{r}} / 2}$; the pair density $\left|\Delta_{\mathbf{r}}\right|^{2}$ equals half the fermion density $\rho_{\mathbf{r}}$, and $\Delta_{\mathbf{r}}=\left|\Delta_{\mathbf{r}}\right| e^{i \theta_{\mathbf{r}}}$ coincides with the bosonic field $\psi_{\mathbf{r}}=\sqrt{\rho_{\mathbf{r}} / 2} e^{i \theta_{\mathbf{r}}}$. To order $\mathcal{O}\left(\rho_{\mathbf{r}}^{2}\right)$, we deduce from (31, 33)

$$
\begin{aligned}
S[\rho, \theta]= & \int_{0}^{\beta} d \tau\left\{\sum_{\mathbf{r}}\left[\frac{i}{2} \rho_{\mathbf{r}} \dot{\theta}_{\mathbf{r}}-\left(h_{0}+\frac{J z}{4}\right) \rho_{\mathbf{r}}\right]\right. \\
& +\frac{J}{4} \sum_{\left\langle\mathbf{r}, \mathbf{r}^{\prime}\right\rangle}\left[\rho_{\mathbf{r}} \rho_{\mathbf{r}^{\prime}}\right. \\
& \left.\left.-\left(\rho_{\mathbf{r}} \rho_{\mathbf{r}^{\prime}}\right)^{1 / 2}\left(2-\rho_{\mathbf{r}}\right) \cos \left(\theta_{\mathbf{r}}-\theta_{\mathbf{r}^{\prime}}\right)\right]\right\}
\end{aligned}
$$

The term $(i / 2) \rho_{\mathbf{r}} \dot{\theta}_{\mathbf{r}}$ comes from the Berry phase term $\left\langle\boldsymbol{\Omega}_{\mathbf{r}} \mid \dot{\boldsymbol{\Omega}}_{\mathbf{r}}\right\rangle$ of the action $S[\boldsymbol{\Omega}]$ [Eq. (31)] with a proper gauge choice $^{20}$ If we further assume that $\rho_{\mathbf{r}}$ and $\theta_{\mathbf{r}}$ are slowly varying in space, we obtain

$$
\begin{aligned}
S[\rho, \theta]= & \int_{0}^{\beta} d \tau\left\{\sum_{\mathbf{r}}\left[\frac{i}{2} \rho_{\mathbf{r}} \dot{\theta}_{\mathbf{r}}-\left(h_{0}+\frac{J z}{4}\right) \rho_{\mathbf{r}}+\frac{J z}{4} \rho_{\mathbf{r}}^{2}\right]\right. \\
& \left.-\frac{J}{2} \sum_{\left\langle\mathbf{r}, \mathbf{r}^{\prime}\right\rangle}\left(\rho_{\mathbf{r}} \rho_{\mathbf{r}^{\prime}}\right)^{1 / 2} \cos \left(\theta_{\mathbf{r}}-\theta_{\mathbf{r}^{\prime}}\right)\right\} .
\end{aligned}
$$


or, equivalently,

$$
\begin{aligned}
S\left[\psi^{*}, \psi\right]= & \int_{0}^{\beta} d \tau\left\{\sum_{\mathbf{r}}\left[\psi_{\mathbf{r}}^{*}\left(\partial_{\tau}-\mu_{B}\right) \psi_{\mathbf{r}}+\frac{U_{B}}{2}\left|\psi_{\mathbf{r}}^{4}\right|\right]\right. \\
& \left.-t_{B} \sum_{\left\langle\mathbf{r}, \mathbf{r}^{\prime}\right\rangle}\left(\psi_{\mathbf{r}}^{*} \psi_{\mathbf{r}^{\prime}}+\text { c.c. }\right)\right\},
\end{aligned}
$$

where $t_{B}=J / 2, U_{B}=2 J z, \mu_{B}=2 h_{0}+J z / 2$, and $z$ is the number of nearest-neighbor sites. We therefore obtain the action of the Bose-Hubbard model with on-site repulsive interaction $U_{B}$ and nearest-neighbor hopping amplitude $t_{B}$. In the continuum limit and for a cubic lattice, the latter gives a boson mass $m_{B}=1 / J$ as obtained in Ref. 20.

\section{CONCLUDING REMARKS}

In this paper, we have shown that a Fermi superfluid in the strong-coupling limit, where superfluidity originates from $\mathrm{BEC}$ of composite bosons, can be described by the complex field $\psi_{\mathbf{r}}=\sqrt{\rho_{\mathbf{r}} / 2} e^{i \theta_{\mathbf{r}}}$, where $\rho_{\mathbf{r}}$ is the fermion density and $\theta_{\mathbf{r}}$ the phase of the pairing field $\Delta_{\mathbf{r}}$. Such is description is made possible by the fact that density $\left(\rho_{\mathbf{r}}\right)$ and amplitude $\left(\left|\Delta_{\mathbf{r}}\right|\right)$ fluctuations are not independent in the strong-coupling limit. The effective action $S[\rho, \theta]$ is derived by introducing the physical fields $\rho_{\mathbf{r}}$ and $\Delta_{\mathbf{r}}$ from the outset by means of Lagrange multiplier fields $\rho_{\mathbf{r}}^{\mathrm{HS}}$ and $\Delta_{\mathbf{r}}^{\mathrm{HS}}$. The latter play the role of the Hubbard-Stratonovich fields usually introduced via a Hubbard-Stratonovich transformation of the fermionfermion interaction.

For continuum models, the effective action is derived from an expansion about the mean-field state. It corresponds to the usual action of a Bose superfluid of density $\rho_{\mathbf{r}} / 2$ where the bosons have a mass $m_{B}=2 m$ and interact via a contact potential with amplitude $g_{B}=$ $4 \pi a_{B} / m_{B}, a_{B}=2 a$.

For lattice (Hubbard) models, the effective action is obtained from an expansion about the $t=0$ limit, using the mapping of the attractive Hubbard model in the strongcoupling limit onto the Heisenberg model in a uniform magnetic field. The effective model is a Bose-Hubbard model with an on-site repulsion $U_{B}=2 J z$ (with $z$ the number of nearest-neighbor sites) and a nearest-neighbor intersite hopping amplitude $t_{B}=J / 2$, where $J=4 t^{2} / U$.

\section{APPENDIX A: LOW-ENERGY EFFECTIVE ACTION $S[\rho, \Delta]$}

In this appendix, we derive the effective action (17) starting from Eq. (16). The first and second order cumulants are given by

$$
\begin{aligned}
\left\langle S^{\prime}\right\rangle= & \int_{0}^{\beta} d \tau \int d^{3} \mathbf{r}\left[-i \rho_{0} \rho_{\mathbf{r}}^{\mathrm{HS}}-i \Delta_{0}\left(\Delta_{\mathbf{r}}^{\mathrm{HS}}+\Delta_{\mathbf{r}}^{\mathrm{HS}}\right)\right], \\
\left\langle S^{\prime 2}\right\rangle_{c}= & \sum_{q}\left[-\rho_{-q}^{\mathrm{HS}} \Pi_{00}^{z z}(q) \rho_{q}^{\mathrm{HS}}-2 \rho_{-q}^{\mathrm{HS}} \Pi_{00}^{z+}(q) \Delta_{q}^{\mathrm{HS}}-2 \rho_{-q}^{\mathrm{HS}} \Pi_{00}^{z-}(q) \Delta_{-q}^{\mathrm{HS}}-\Delta_{-q}^{\mathrm{HS}} \Pi_{00}^{++}(q) \Delta_{q}^{\mathrm{HS}}\right. \\
& \left.-\Delta_{q}^{\mathrm{HS}^{*}} \Pi_{00}^{--}(q) \Delta_{-q}^{\mathrm{HS}}-2 \Delta_{-q}^{\mathrm{HS}} \Pi_{00}^{+-}(q) \Delta_{-q}^{\mathrm{HS}}\right] .
\end{aligned}
$$

The second order cumulant is written in Fourier space. The mean-field correlation function $\Pi_{00}^{\nu \nu^{\prime}}(q)=\left\langle j_{0}^{\nu}(q) j_{0}^{\nu^{\prime}}(-q)\right\rangle_{c}$ is defined in Sec. IIB. To obtain (A1), we have used the fact that mean-field correlation functions involving the current $\mathbf{j}_{\mathbf{r}}^{0}$ vanish (Appendix $\left.\mathbf{B}\right)$. In the low-energy limit, we can approximate $\Pi_{00}^{\nu \nu^{\prime}}(q)$ by its static limit $\Pi_{00}^{\nu \nu^{\prime}}(\mathbf{q})=$ $\Pi_{00}^{\nu \nu^{\prime}}\left(\mathbf{q}, \omega_{\nu}=0\right)$.

Integrating out the Hubbard-Stratonovich field $\rho_{\mathbf{r}}^{\mathrm{HS}}$, we obtain

$$
\begin{aligned}
S= & \int_{0}^{\beta} d \tau \int d^{3} \mathbf{r}\left[-g \delta\left|\Delta_{\mathbf{r}}\right|^{2}-\frac{g}{4} \delta \rho_{\mathbf{r}}^{2}+\rho_{\mathbf{r}}\left(\frac{i}{2} \dot{\theta}_{\mathbf{r}}+\frac{\left(\boldsymbol{\nabla} \theta_{\mathbf{r}}\right)^{2}}{8 m}-\frac{\mu_{B}}{2}\right)+i\left(\Delta_{\mathbf{r}}^{\mathrm{HS}}+\Delta_{\mathbf{r}}^{\mathrm{HS}}\right) \delta\left|\Delta_{\mathbf{r}}\right|\right] \\
& +\sum_{q}\left\{\frac{1}{2} \Pi_{00}^{++}(\mathbf{q})\left(\Delta_{-q}^{\mathrm{HS}} \Delta_{q}^{\mathrm{HS}}+\text { c.c. }\right)+\Delta_{q}^{\mathrm{HS}{ }^{*}} \Pi_{00}^{+-}(\mathbf{q}) \Delta_{q}^{\mathrm{HS}}\right. \\
& \left.+\frac{1}{2} \Pi_{00}^{z z}(\mathbf{q})^{-1}\left[\delta \rho_{-q} \delta \rho_{q}-2 i \Pi_{00}^{z+}(q) \delta \rho_{-q}\left(\Delta_{q}^{\mathrm{HS}}+\Delta_{-q}^{\mathrm{HS}}\right)-\Pi_{00}^{z+}(\mathbf{q})^{2}\left(\Delta_{-q}^{\mathrm{HS}} \Delta_{q}^{\mathrm{HS}}+\Delta_{-q}^{\mathrm{HS}} \Delta_{q}^{\mathrm{HS}}+2\left|\Delta_{q}^{\mathrm{HS}}\right|^{2}\right)\right]\right\},(\mathrm{A}
\end{aligned}
$$

where $\delta \rho_{\mathbf{r}}=\rho_{\mathbf{r}}-\rho_{0}$ and $\delta\left|\Delta_{\mathbf{r}}\right|=\Delta_{\mathbf{r}}-\Delta_{0}$. Here we have neglected constant terms and use the saddle-point equations (5).

To obtain the action $S[\rho, \Delta]$ in terms of the physical fields only, one has then to integrate out the Hubbard- 
Stratonovich field $\Delta^{\mathrm{HS}}$ :

$$
\begin{aligned}
& \int \mathcal{D}\left[\Delta^{\mathrm{HS}}\right] \exp \left\{-S_{0}\left[\Delta^{\mathrm{HS}}\right]-i \sum_{q}\left(\Delta_{q}^{\mathrm{HS}}+\Delta_{-q}^{\mathrm{HS} *}\right)\left[\delta|\Delta|_{-q}-\Pi_{00}^{z z}(\mathbf{q})^{-1} \Pi_{00}^{z+}(\mathbf{q}) \delta \rho_{-q}\right]\right\} \\
= & \exp \left\{-\frac{1}{2} \sum_{q}\left\langle\left(\Delta_{q}^{\mathrm{HS}}+\Delta_{-q}^{\mathrm{HS}}\right)\left(\Delta_{-q}^{\mathrm{HS}}+\Delta_{q}^{\mathrm{HS}}\right)\right\rangle_{0}\left[\delta|\Delta|_{q}-\Pi_{00}^{z z}(\mathbf{q})^{-1} \Pi_{00}^{z+}(\mathbf{q}) \delta \rho_{q}\right]\left[\delta|\Delta|_{-q}-\Pi_{00}^{z z}(\mathbf{q})^{-1} \Pi_{00}^{z+}(\mathbf{q}) \delta \rho_{-q}\right]\right\} \\
= & \exp \left\{\frac{1}{2} \sum_{q}\left[M_{11}(\mathbf{q})+M_{22}(\mathbf{q})+2 M_{12}(\mathbf{q})\right]\left[\delta|\Delta|_{q}-\Pi_{00}^{z z}(\mathbf{q})^{-1} \Pi_{00}^{z+}(\mathbf{q}) \delta \rho_{q}\right]\left[\delta|\Delta|_{-q}-\Pi_{00}^{z z}(\mathbf{q})^{-1} \Pi_{00}^{z+}(\mathbf{q}) \delta \rho_{-q}\right]\right\},(\mathrm{A})
\end{aligned}
$$

where averages $\langle\cdots\rangle_{0}$ are taken with the Gaussian action

$$
\begin{aligned}
& S_{0}\left[\Delta^{\mathrm{HS}}\right]=-\frac{1}{2} \sum_{q}\left(\Delta_{q}^{\mathrm{HS}}, \Delta_{-q}^{\mathrm{HS}}\right) M^{-1}(\mathbf{q})\left(\begin{array}{c}
\Delta_{q}^{\mathrm{HS}} \\
\Delta_{-q}^{\mathrm{HS}}
\end{array}\right) \\
& M^{-1}(\mathbf{q})=\left(\begin{array}{ll}
-\Pi_{00}^{+-}(\mathbf{q})+\Pi_{00}^{z z}(\mathbf{q})^{-1} \Pi_{00}^{z+}(\mathbf{q})^{2} & -\Pi_{00}^{++}(\mathbf{q})+\Pi_{00}^{z z}(\mathbf{q})^{-1} \Pi_{00}^{z+}(\mathbf{q})^{2} \\
-\Pi_{00}^{++}(\mathbf{q})+\Pi_{00}^{z z}(\mathbf{q})^{-1} \Pi_{00}^{z+}(\mathbf{q})^{2} & -\Pi_{00}^{+-}(\mathbf{q})+\Pi_{00}^{z z}(\mathbf{q})^{-1} \Pi_{00}^{z+}(\mathbf{q})^{2}
\end{array}\right) .
\end{aligned}
$$

In the following, we denote by $A_{\mathbf{q}}$ and $B_{\mathbf{q}}$ the diagonal and off-diagonal components of $M^{-1}(\mathbf{q})$, and $C_{\mathbf{q}}=A_{\mathbf{q}}+B_{\mathbf{q}}$. The effective action $S[\rho, \Delta]$ deduced from Eqs. (A2A3) is given by Eq. (17).

\section{APPENDIX B: MEAN-FIELD CORRELATION FUNCTION}

In this appendix, we calculate the mean-field correlation function $\Pi_{\mu \mu^{\prime}}^{\nu \nu^{\prime}}(q)=\left\langle j_{\mu}^{\nu}(q) j_{\mu^{\prime}}^{\nu^{\prime}}(-q)\right\rangle_{c}\left(\nu, \nu^{\prime}=x, y, z\right.$; $\left.\mu, \mu^{\prime}=0, x, y, z\right)$ in the strong-coupling limit $\rho_{0} a^{3} \ll 1$ and for $|\mathbf{q}| a \ll 1$.

\section{General expression}

$j_{\mu}^{\nu}(q)$ is the Fourier transformed field of $j_{\mu \mathbf{r}}^{\nu}$ [Eq. [15]]:

$$
\begin{aligned}
& j_{0}^{\nu}(q)=\frac{1}{\sqrt{\beta V}} \sum_{k} \phi_{k}^{\dagger} \sigma^{\nu} \phi_{k+q}, \\
& j_{\mu}^{0}(q)=\frac{1}{\sqrt{\beta V}} \sum_{k} \frac{1}{m}\left(k_{\mu}+\frac{q_{\mu}}{2}\right) \phi_{k}^{\dagger} \phi_{k+q}(\mu \neq 0)(\mathrm{B} 1)
\end{aligned}
$$

where $V$ is the volume of the system and $V^{-1} \sum_{\mathbf{k}}=\int_{\mathbf{k}}$ for $V \rightarrow \infty . \quad k=(\mathbf{k}, i \omega)$ and $\sum_{k}=\sum_{\mathbf{k}, \omega}$ where $\omega$ is a fermionic Matsubara frequency. We have

$$
\begin{aligned}
\Pi_{00}^{z z}(q)= & -\frac{2}{\beta V} \sum_{k}[G(k) G(k+q)-F(k) F(k+q)], \\
\Pi_{00}^{z+}(q)= & \Pi_{00}^{z-}(-q)=-\frac{2}{\beta V} \sum_{k} G(k+q) F(k), \\
\Pi_{00}^{++}(q)= & \Pi_{00}^{--}(q)=-\frac{1}{\beta V} \sum_{k} F(k) F(k+q), \\
\Pi_{00}^{+-}(q)= & \frac{1}{\beta V} \sum_{k} G(k) G(-k-q), \\
\Pi_{\mu \mu^{\prime}}^{00}(q)= & -\frac{2}{\beta V} \sum_{k} \frac{1}{m^{2}}\left(k_{\mu}+\frac{q_{\mu}}{2}\right)\left(k_{\mu^{\prime}}+\frac{q_{\mu^{\prime}}}{2}\right) \\
& \times[G(k+q) G(k)+F(k+q) F(k)],
\end{aligned}
$$

where $G$ and $F$ are the mean-field propagators [Eq. (7)]. The correlation function $\left\langle j_{\mu}^{0}(q) j_{0}^{\nu}(-q)\right\rangle(\mu \neq 0)$ vanishes.

In the following, we consider the static limit $\Pi_{00}^{\nu \nu^{\prime}}(\mathbf{q})=$ $\Pi_{00}^{\nu \nu^{\prime}}\left(\mathbf{q}, \omega_{\nu}=0\right)$. Performing the sum over Matsubara frequency in (B2) in the $T=0$ limit, we obtain

$$
\begin{aligned}
\Pi_{00}^{z z}(\mathbf{q}) & =\int_{\mathbf{k}} \frac{1}{E_{\mathbf{k}}+E_{\mathbf{k}+\mathbf{q}}}\left(1-\frac{\xi_{\mathbf{k}} \xi_{\mathbf{k}+\mathbf{q}}}{E_{\mathbf{k}} E_{\mathbf{k}+\mathbf{q}}}+\frac{\Delta_{0}^{\mathrm{HS}}{ }^{2}}{E_{\mathbf{k}} E_{\mathbf{k}+\mathbf{q}}}\right), \\
\Pi_{00}^{z+}(\mathbf{q}) & =\Pi_{00}^{z-}(\mathbf{q})=\int_{\mathbf{k}} \frac{\Delta_{0}^{\mathrm{HS}} \xi_{\mathbf{k}}}{\left(E_{\mathbf{k}}+E_{\mathbf{k}+\mathbf{q}}\right) E_{\mathbf{k}} E_{\mathbf{k}+\mathbf{q}}}, \\
\Pi_{00}^{++}(\mathbf{q}) & =\Pi_{00}^{--}(\mathbf{q})=-\int_{\mathbf{k}} \frac{\Delta_{0}^{\mathrm{HS}^{2}}}{2\left(E_{\mathbf{k}}+E_{\mathbf{k}+\mathbf{q}}\right) E_{\mathbf{k}} E_{\mathbf{k}+\mathbf{q}}}, \\
\Pi_{00}^{+-}(\mathbf{q}) & =\int_{\mathbf{k}} \frac{1}{2\left(E_{\mathbf{k}}+E_{\mathbf{k}+\mathbf{q}}\right)}\left(1+\frac{\xi_{\mathbf{k}} \xi_{\mathbf{k}+\mathbf{q}}}{E_{\mathbf{k}} E_{\mathbf{k}+\mathbf{q}}}\right) .
\end{aligned}
$$

The correlation function $\Pi_{\mu \mu^{\prime}}^{00}(\mathbf{q})$ vanishes for $\mathbf{q}=0$. Since $j_{\mu \mathbf{r}}^{0}$ multiplies $\partial_{\mu} \theta_{\mathbf{r}}$ in the action $S^{\prime}$, it is sufficient 
to consider $\Pi_{\mu \mu^{\prime}}^{00}(\mathbf{q}=0)$ to obtain the effective action $S[\rho, \Delta]$ to order $\left(\partial_{\mu} \theta_{\mathbf{r}}\right)^{2}$.

We next expand the correlations to order $\mathcal{O}\left(|\mathbf{q}|^{2}\right)$. Writing $\xi_{\mathbf{k}+\mathbf{q}}=\xi_{\mathbf{k}}+X_{\mathbf{k}, \mathbf{q}}$ with $X_{\mathbf{k}, \mathbf{q}}=\mathbf{k} \cdot \mathbf{q} / m+|\mathbf{q}|^{2} / 2 m$, we obtain

$$
\begin{aligned}
& \Pi_{00}^{z z}(\mathbf{q})= \int_{\mathbf{k}}\left[\frac{\Delta_{0}^{H S^{2}}}{E_{\mathbf{k}}^{3}}+\left(-\frac{3 \xi_{\mathbf{k}}}{2 E_{\mathbf{k}}^{3}}+\frac{3 \xi_{\mathbf{k}}^{3}}{2 E_{\mathbf{k}}^{5}}\right) X_{\mathbf{k}, \mathbf{q}}\right. \\
&\left.+\left(-\frac{1}{2 E_{\mathbf{k}}^{3}}+\frac{3 \xi_{\mathbf{k}}^{2}}{E_{\mathbf{k}}^{5}}-\frac{5 \xi_{\mathbf{k}}^{4}}{2 E_{\mathbf{k}}^{7}}\right) X_{\mathbf{k}, \mathbf{q}}^{2}\right] \\
& \Pi_{00}^{z \pm}(\mathbf{q})= \int_{\mathbf{k}} \frac{\Delta_{0}^{\mathrm{HS}} \xi_{\mathbf{k}}}{2 E_{\mathbf{k}}^{3}}\left(1-\frac{3 \xi_{\mathbf{k}}}{2 E_{\mathbf{k}}^{2}} X_{\mathbf{k}, \mathbf{q}}\right. \\
&\left.-\frac{3 E_{\mathbf{k}}^{2}-10 \xi_{\mathbf{k}}^{2}}{4 E_{\mathbf{k}}^{4}} X_{\mathbf{k}, \mathbf{q}}^{2}\right) \\
& \Pi_{00}^{++}(\mathbf{q})= \Pi_{00}^{--}(\mathbf{q}) \\
&-\int_{\mathbf{k}} \frac{\Delta_{0}^{\mathrm{HS}}}{4 E_{\mathbf{k}}^{3}}\left(1-\frac{3 \xi_{\mathbf{k}}}{2 E_{\mathbf{k}}^{2}} X_{\mathbf{k}, \mathbf{q}}\right. \\
&\left.-\frac{3 E_{\mathbf{k}}^{2}-10 \xi_{\mathbf{k}}^{2}}{4 E_{\mathbf{k}}^{2}} X_{\mathbf{k}, \mathbf{q}}^{2}\right) \\
& \frac{1}{2} \int_{\mathbf{k}}\left[\frac{1}{2 E_{\mathbf{k}}}+\frac{\xi_{\mathbf{k}}^{2}}{2 E_{\mathbf{k}}^{3}}+\left(\frac{\xi_{\mathbf{k}}}{4 E_{\mathbf{k}}^{3}}-\frac{3 \xi_{\mathbf{k}}^{3}}{4 E_{\mathbf{k}}^{5}}\right) X_{\mathbf{k}, \mathbf{q}}\right. \\
&\left.+\left(-\frac{1}{8 E_{\mathbf{k}}^{3}}-\frac{7 \xi_{\mathbf{k}}^{2}}{8 E_{\mathbf{k}}^{5}}+\frac{5 \xi_{\mathbf{k}}^{4}}{4 E_{\mathbf{k}}^{7}}\right) X_{\mathbf{k}, \mathbf{q}}^{2}\right] \\
& \Pi_{00}^{+-}(\mathbf{q})
\end{aligned}
$$

\section{Strong-coupling limit $\rho_{0} a^{3} \ll 1$}

In the strong-coupling limit, the chemical potential $\mu$ is negative. We then have

$$
\begin{aligned}
& \int_{\mathbf{k}} \frac{1}{\xi_{\mathbf{k}}}=\frac{m \Lambda}{\pi^{2}}-\frac{m^{3 / 2}|\mu|^{1 / 2}}{\sqrt{2} \pi} \\
& \int_{0}^{\infty} d k \frac{k^{4}}{\xi_{\mathbf{k}}^{3}}=\frac{3 \pi m^{5 / 2}}{2 \sqrt{2}|\mu|^{1 / 2}}
\end{aligned}
$$

Other useful relations are obtained by differentiating Eqs. (B5) with respect to $\mu$. Note that $\Lambda$ is sent to infinity whenever the integral over $\mathbf{k}$ converges. In the strong-coupling limit, the small parameter expansion is $\Delta_{0}^{\mathrm{HS}^{2}} /|\mu|^{2} \sim \rho_{0} a^{3}$. Approximate expressions of the mean-field correlation functions can be obtained by expanding Eqs. (8) and (B4) in power of $\Delta_{0}^{\mathrm{HS}^{2}}$ and using Eqs. (B5) (as well as those obtained from (B5) by differentiating with respect to $\mu$ ). A straightforward (but somewhat lengthly) calculation then gives Eq. (9) and

$$
\begin{aligned}
\Pi_{00}^{z z}(\mathbf{q}) & =\rho_{0} m a^{2}\left(1-4 \pi \rho_{0} a^{3}-\frac{|\mathbf{q}|^{2} a^{2}}{4}\right) \\
\Pi_{00}^{z \pm}(\mathbf{q}) & =\left(\frac{\rho_{0} m^{2} a}{4 \pi}\right)^{1 / 2}\left(1-\frac{7}{4} \pi \rho_{0} a^{3}-\frac{|\mathbf{q}|^{2} a^{2}}{12}\right), \\
\Pi_{00}^{++}(\mathbf{q}) & =-\frac{\rho_{0} m a^{2}}{4}\left(1-4 \pi \rho_{0} a^{3}-\frac{5}{16}|\mathbf{q}|^{2} a^{2}\right) \\
\Pi_{00}^{+-}(\mathbf{q}) & =\frac{1}{g}-\frac{\rho_{0} m a^{2}}{4}\left(1-4 \pi \rho_{0} a^{3}\right)-\frac{m a|\mathbf{q}|^{2}}{32 \pi} .
\end{aligned}
$$

1 J. Kinast, S.L. Hemmer, M.E. Gehm, A. Turpalov, and J.E. Thomas, Phys. Rev. Lett. 92, 150402 (2004).

${ }^{2}$ M. Bartenstein, A. Altmeyer, S. Riedl, S. Joachim, C. Chin, J.H. Denschlag, and R. Grimm, Phys. Rev. Lett. 92, 120401 (2004).

3 M.W. Zwierlein, C.A. Stan, C.H. Schunk, S.M.F. Raupach, A.J. Kerman, and W. Ketterle, Phys. Rev. Lett. 92, 120403 (2004).

4 C.A. Regal, M. Greiner, and D.S. Jin, Phys. Rev. Lett. 92, 040403 (2004).

5 T. Bourdel, L. Khaykovich, J. Cubizolles, J. Zhang, F. Chevy, M. Teichmann, L. Tarruell, S. J. J. M. F. Kokkelmans, and C. Salomon, Phys. Rev. Lett. 93, 050401 (2004).

6 For a review on the BCS-Bose crossover in Fermi superfluids, see M. Randeria in Bose-Einstein condensation, edited by A. Griffin, D.W. Snoke, and S. Stringari (Cambridge University Press, 1995).

7 For earlier works on the BCS-Bose crossover in Fermi su- perfluids, see D.M. Eagles, Phys. Rev. 186, 456 (1969); A.J. Leggett in Modern Trends in the Theory of Condensed Matter, edited by A. Peralski and J. Przystawa (SpringerVerlag, Berlin, 1980); P. Nozières and S. Schmitt-Rink, J. Low Temp. Phys. 59, 195 (1985).

8 E.P. Gross, Nuovo Cimento 20, 454 (1961).

${ }^{9}$ L.P. Pitaevskii, Sov. Phys. JETP 12, 155 (1961).

10 R.P. Feynman, Statistical Mechanics (Benjamin, 1972).

11 P. Ao, D.J. Thouless, and X.M. Zhu, Mod. Phys. Lett. B 9, 755 (1995).

12 See, for instance, L. Pitaevskii and S. Stringari, BoseEinstein condensation (Oxford University Press, 2003).

13 M. Drechsler and W. Zwerger, Ann. Physik 1, 15 (1992).

14 R. Haussmann, Z. Phys. 91, 291 (1993).

15 C. A. R. Sá de Melo, M. Randeria, and J. R. Engelbrecht, Phys. Rev. Lett. 71, 3202 (1993); J. R. Engelbrecht, M. Randeria, and C. A. R. Sá de Melo, Phys. Rev. B 55, 15153 (1997). 
16 F. Pistolesi and G.C. Strinati, Phys. Rev. B 53, 15168 (1996).

17 P. Pieri and G.C. Strinati, Phys. Rev. B 61, 15370 (2000).

18 P. Pieri and G.C. Strinati, Phys. Rev. Lett. 91, 030401 (2003).

19 S. De Palo, C. Castellani, C. Di Castro, and B.K. Chakraverty, Phys. Rev. B 60, 564 (1999).

20 N. Dupuis, Phys. Rev. B 70, 134502 (2004).

21 See also M. Stone, Int. J. Mod. Phys. B 9, 1359 (1995); I.J.R Aitchison, P. Ao, D.J. Thouless, and X.M. Zhu, Phys. Rev. B 51, 6531 (1995).

22 See, for instance, M. Holland, S.J.J.M.F. Kokkelmans, M.L. Chiofalo, and R. Walser, Phys. Rev. Lett. 87, 120406 (2001); Y. Ohashi and A. Griffin, Phys. Rev. Lett. 89, 130402 (2002).

${ }^{23}$ G.M. Falco and H.T.C. Stoof, Phys. Rev. Lett. 92, 130401 (2004).

24 S. Simonucci, P. Pieri, and G.C. Strinati, Europhys. Lett. 69, 713 (2005).

${ }^{25}$ Here we use the identity $c_{\mathbf{r} \uparrow}^{*} c_{\mathbf{r} \downarrow}^{*} c_{\mathbf{r} \downarrow} c_{\mathbf{r} \uparrow}=\frac{1}{4}\left[\left(c_{\mathbf{r}}^{\dagger} c_{\mathbf{r}}\right)^{2}-\right.$ $\left.\left(c_{\mathbf{r}}^{\dagger} \sigma^{z} c_{\mathbf{r}}\right)^{2}\right]$.

26 An opposite sign for the coefficient of the $\delta \rho_{-q} \delta|\Delta|_{q}$ would give a minus sign in Eq. (21), i.e. $\delta\left|\Delta_{\mathbf{r}}\right| \delta \rho_{\mathbf{r}}<0$. Since $\left|\Delta_{\mathbf{r}}\right|^{2}$ is related to the density of Cooper pairs, we expect $\left|\Delta_{\mathbf{r}}\right|$ to increase with the fermion density, i.e. $\delta\left|\Delta_{\mathbf{r}}\right| \delta \rho_{\mathbf{r}}>0$, in agreement with Eq. 21). Note also that a minus sign in Eq. (21) would not allow us to recover the bosonic action (24).

27 Mean-field correlation functions and their strong-coupling limit have also been computed in M. Marini, F. Pistolesi, and G.C. Strinati, Eur. Phys. J. B 1, 151 (1998).

28 D.S. Petrov, C. Salomon, and G.V. Shlyapnikov, Phys. Rev. Lett. 93, 090404 (2004).
${ }^{29}$ In Ref. 19, it was (incorrectly) stated that the coefficient $1 / 32$ in the term $\left(\nabla \rho_{\mathbf{r}}\right)^{2} / 32 \rho_{0} m$ is not obtained from the expansion about the mean-field state, so that the action (24) is recovered only in the strict hydrodynamic limit where only the gradient term $\rho_{\mathbf{r}}\left(\nabla \theta_{\mathbf{r}}\right)^{2} / 8 m$ contained in $\left|\nabla \psi_{\mathbf{r}}\right|^{2} / 4 m$ is retained, while the term $\left(\nabla \rho_{\mathbf{r}}\right)^{2} / 32 m \rho_{\mathbf{r}}$ is ignored.

30 In our formalism, this approach amounts to integrating out the physical field $\Delta_{\mathrm{r}}$ from the outset and then carrying out the expansion about the non-interacting state. Integrating out the fermions and $\rho_{\mathbf{r}}^{\mathrm{HS}}$, one obtains $\left|\Delta_{\mathbf{r}}^{\mathrm{HS}}\right|=$ $\left(4 \pi \rho_{\mathbf{r}} / m^{2} a\right)^{1 / 2}$ and an effective action $S\left[\rho_{\mathbf{r}}, \theta_{\mathbf{r}}^{\mathrm{HS}}\right]$, where $\theta_{\mathbf{r}}^{\mathrm{HS}}$ is the phase of the Hubbard-Stratonovich field $\Delta_{\mathbf{r}}^{\mathrm{HS}}$. When written in terms of the field $\sqrt{\rho_{\mathbf{r}} / 2} e^{i \theta_{\mathbf{r}}^{\mathrm{HS}}}$, the action takes the form (24).

31 The derivation of the effective action based on the mapping of the attractive Hubbard model onto the repulsive Hubbard model presents several interesting features. First, in the repulsive model, the difficulties with dealing with both the particle-particle and particle-hole channels do not arise since all the important fluctuations pertain to the particlehole channel. Second, the repulsive model allows one to take into account on the same footing $\mathbf{q}=(\pi, \pi)$ density and $\mathbf{q}=0$ pairing fluctuations near half-filling. At halffilling, both types of fluctuations are degenerate so that the symmetry of the order parameter is enlarged to $\mathrm{SO}(3)$. See Ref. 20 for a detailed discussion.

32 R. Micnas, J. Ranninger, and S. Robaszkiewicz, Rev. Mod. Phys. 62, 113 (1990).

33 See, for instance, A. Auerbach, Interacting Electrons and Quantum Magnetism (Springer-Verlag, New York, 1994). 